\title{
36. OBSERVATIONS OF METEORIC DUST FROM ROCKETS AND SATELLITES
}

\author{
(Survey Paper) \\ T. N. Nazarova \\ (Academy of Sciences of the U.S.S.R., Moscow)
}

18 years have elapsed since the first data on meteoritic dust were obtained by means of the instruments installed on a rocket under the guidance of Professor Bohn. Since then these investigations, by means of different instrumentation, have been carried on in the vicinity of the Earth and in interplanetary space. By now rocket and satellite data have been obtained on the spatial density of meteoritic particles with masses of $10^{-7}-10^{-16}$ grams and on their energy; a concept of the mass spectrum of these particles has been obtained as well as the first data on their velocities. Over recent years very difficult experiments have been initiated for obtaining samples of meteoritic particles directly from high altitudes with a view to determining their physical properties, chemical composition, and the destroying effect.

Hemenway et al. (1967) have compiled a summary graph representing data obtained by different methods and at different distances from the Earth.

However, before commenting on these data I should like to say a few words about the degree of reliability of measurements carried out by means of acoustical sensors since these sensors are widely used in flights and their use, as was pointed out recently, involves serious drawbacks.

In the first place, acoustical sensors are subject to the influence of temperature gradients which results in many false signals.

Secondly, sensor calibration contains many uncertainties and, consequently, the mass spectrum obtained from measurements may be incorrect.

Thirdly, it is probable that acoustical sensors give false signals under the effect of the dust 'atmosphere' of a space probe or a satellite.

As all other investigators, we naturally desire to have only reliable data. Therefore, after the publication of Nilsson's (1966) paper on the influence of low-temperature gradients on his acoustical sensors, we have conducted similar experiments. Earlier our acoustical sensors were tested as regards the influence of temperature over a large temperature range. Recently we have conducted laboratory investigations on the effect on our sensors of the actual temperature gradients which this or that space vehicle experiences in flight. Testing of a detector was carried out in the temperature interval from $50^{\circ} \mathrm{C}$ below zero to $100^{\circ} \mathrm{C}$ above zero, with positive and negative tem-

Kresák and Millman (eds.), Physics and Dynamics of Meteors, 382-387. C I.A.U. 
perature gradients from $0.02^{\circ}$ to $0.5^{\circ}$ per minute, as it was in flights. In addition, the influence of positive and negative gradients of the order of a few degrees was tested.

Experiments have demonstrated that under the effect of temperature gradients the sensor did not give false signals. The analysis of the data of our flights also confirms the lack of a correlation between the temperature gradients and the number of signals given by the sensors.

This gives ground to believe that there were no false signals in our flight experiments, except maybe for some single ones caused by an unknown reason. These will not change the final result, if one takes into account the accuracy of space-density estimates which are available.

Negative results concerning the influence of the temperature gradients on ceramic sensors used in 11 American flight experiments, on the basis of laboratory tests and analysis of flight data, were reported by Bohn et al. (1967a) at the COSPAR Symposium in July.

Let us turn now to the problem of sensor calibration:

For interpreting our experimental data we have used the theoretical dependence of a pulse recorded by the sensor on the energy of a particle. Bohn et al. (1967b) have obtained experimentally for ceramic sensors the dependence $S \sim m v^{\alpha}$, where $\alpha \geqslant 2$. It should be mentioned that the change in calibration has resulted in a shift of the particle mass-distribution curve by approximately half an order of magnitude.

Since, when deciphering data, a velocity value should be assumed, we naturally obtain only a concept of the mass spectrum, and in this sense the criticism concerning acoustical sensors (as in many other cases) is correct. The last comment concerns the possibility of recording the dust 'atmosphere' of a rocket or a satellite by acoustical sensors.

The particles constituting the dust atmosphere of a space vehicle have very low velocities with respect to it, of the order of centimetres per second. Acoustical sensors can record them if the particles of the vehicle 'atmosphere' have large masses. However, it is difficult to imagine that there will be many large particles causing false signals, and that they will be able to follow the vehicle during a very large period of time (up to a few months) causing false registrations, non-uniform in time, sometimes with large intervals of several weeks.

Hence, we regard our data obtained by means of acoustical sensors as sufficiently reliable, and at least as accurate as all rechecked U.S. experiments, in which sensors of such type were used, and experiments conducted by Wlochowicz (1966).

Let us now return to the graph which represents data on the study of space dust by means of rockets and satellites.

While considering this it should be kept in mind that different methods of recording particles are based on different physical phenomena which accompany the impact of fast-flying meteoritic particles (with all their peculiarities) against an obstacle, and this may lead to some differences in the final results. In our opinion, this is a probable reason for the disagreement between data obtained by acoustical methods and 


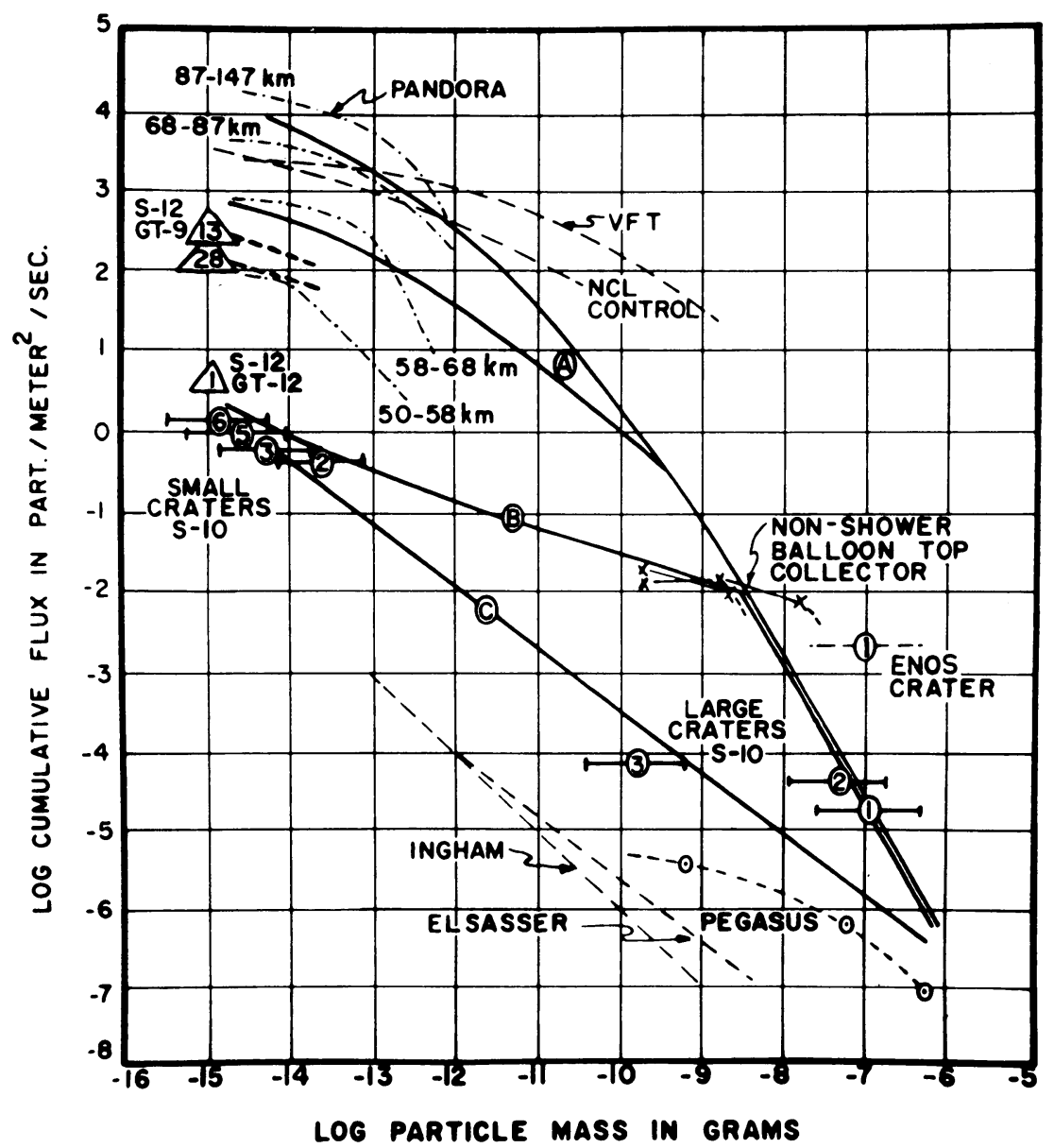

Fig. 1. Cumulative flux distribution based on data from various sources. (Courtesy of C. L. Hemenway.)

methods based on the penetration of a meteoritic body into the obstacle. Of course, during comparison of data one should take into account the distances from Earth at which the experiment was performed, as well as the conditions under which it was made, and the size of sensitive areas of the detectors.

As is evident from Figure 1, there is a large amount of data obtained at altitudes of $60-150 \mathrm{~km}$ for particles with masses $10^{-17}-10^{-11}$ grams.

In our judgment, while analyzing the mass spectrum of meteoritic particles, data from measurements at altitudes of about 100-200 km should be singled out. At such heights both ground-based methods (analysis of brightness of the twilight sky) and rocket measurements have shown a very high concentration of minute dust particles, mainly with low velocities. These particles form the dust halo of the Earth. 
While considering the problem of spatial density of meteoritic bodies at larger distances from the Earth, the Earth itself and its artificial satellites should be considered as a specific probe which moves in interplanetary space and encounters meteoritic showers, individual aggregations and sporadic meteoritic bodies. Thus at large distances mainly fluctuations of the spatial density of meteoritic bodies are observed that reflect the picture of their distribution in space. The Earth's dust envelope, of course, is also subjected to fluctuations but somewhat differently, due to the presence of the dense atmosphere. Measurements from Electron-2 and Electron-4 satellites illustrate this point. These satellites were equipped with identical meteoritic instrumentation and were placed into similar orbits (apogee at about $4000 \mathrm{~km}$, perigee at about $400 \mathrm{~km}$ ) but operated at different times. From January 30 to March 10, 1964, for a total operating time of about 479 hours, Electron-2 recorded three aggregations of meteoritic particles, with intervals between them of a few days, and in addition four particles with great intervals between them. In all 223 particles were recorded.

From July 11 to September 1, 1964, Electron-4, for 898 hours, recorded only nine particle impacts, the time between them varying approximately from a day to a week.

Incidentally, we have not observed the Earth's dust cloud at distances of thousands of kilometres from its surface, predicted on the basis of theoretical work. Perhaps, this is on account of its very low density.

The influence of showers and aggregations, or unknown showers, on the particle concentration in the neighbourhood of the Earth was detected from observations on Sputnik-3, when a particle-density increase by approximately 3 orders of magnitude was recorded. The influence of showers was shown also by Dubin et al. (1963) from data of Vanguard-3, when under the influence of the Leonid shower the particle concentration increased by approximately 2 orders of magnitude. An increase of particle concentration by 2 orders of magnitude under the effect of an unknown shower was also recorded by Explorer-I. Diurnal variations of particle concentration reaching one order of magnitude have been observed.

The curve close to the model B suggested by Hemenway et al. (1967), constructed by taking into account the results of investigations on Gemini S-10, seems to be the most acceptable average curve of particle mass distribution outside the Earth's dust envelope.

By means of Soviet and American space rockets several soundings of interplanetary space were performed along the flight trajectory of rockets from the Earth's orbit towards the Sun and in the opposite direction.

Instrumentation installed on Soviet space probes Mars-1, Zond-3 and Venus- 2 have recorded in interplanetary space the presence of meteoritic particle aggregations whose extent varied within wide limits, reaching millions of kilometres. The long aggregations recorded by the space probes contained individual condensations of meteoritic particles, for which the impact rate per $\mathrm{m}^{2}$ per second varied from $10^{-1}$ to $10^{-5}$.

It should be mentioned that the intervals between individual aggregations, during 
which not a single impact was registered, ranged from a few days to about 1 month. The instrumentation threshold sensitivity was $10^{-7}$ grams. From this it follows that the spatial density of particles with masses $\geqslant 10^{-7}$ grams, of a definite sufficiently long time and in definite regions of space, was lower than $10^{-6}-2 \times 10^{-7}$ particles $\sec ^{-1}$ impacting on $1 \mathrm{~m}^{2}$.

Kramer's theoretical calculations (U.S.S.R.) have shown that the greater part of the aggregations observed on these flights coincided with the intersection points of the ecliptic plane with the orbits of meteor showers and comets.

Measurements from space probes Zond-3 and Venus-2 with a threshold sensitivity of instrumentation $m \geqslant 10^{-7}$ grams for a particle velocity of $15 \mathrm{~km} / \mathrm{sec}$, have shown the lack of any considerable difference in average spatial density of the material toward the Sun and away from the Sun. The average amount of meteoritic matter recorded in these experiments (including showers) in the direction away from the Sun exceeded the amount in the direction to the Sun only by a factor of $1 \cdot 5-2$.

New measurements of the flux of interplanetary dust particles in interplanetary space have been obtained from experiments on Mariner-IV spacecraft.

For the first time Mariner-IV measurement provides two data samples over the same heliocentric distance in the zodiacal dust cloud $(\sim 1.1 \mathrm{AU}$ to $1.25 \mathrm{AU})$ with the same instrumentation. This increases considerably their reliability. In the first case the flux of dust was $7.3 \times 10^{-5}$ particles $/ \mathrm{m}^{2} \mathrm{sec} . \pi$. ster, and in the second case it was $1 \cdot 1 \times 10^{-5}$ particles $/ \mathrm{m}^{2} \mathrm{sec}$. $\pi$. ster, with the instrumentation sensitivity $6 \pm 0.7 \times 10^{-5}$ dyne-sec. The results of Mariner-IV experiments are in agreement with Zond-3 data. The space density of meteoric particles from the Zond-3 experiment, apart from particles belonging to known meteor showers, was $2 \times 10^{-5}$ particles $\mathrm{m}^{-2} \mathrm{sec}^{-1}$ for $m \geqslant 10^{-7} \mathrm{gm}$.

The OGO-III measurement was the only measurement which repeatedly sampled the cis-lunar dust particle flux over a considerable period of time. The bulk of the measurements on OGO-III were performed at distances between $50000 \mathrm{~km}$ and $110000 \mathrm{~km}$ from the Earth's surface. For particles with the mass of $0 \cdot 1-1 \cdot 10^{-9} \mathrm{grams}$ a spatial density of $10^{-2}$ particles $/ \mathrm{m}^{2} \mathrm{sec}$. $\pi$. sterad was recorded. This is in good agreement with the Hemenway ' $B$ ' curve.

The increased density of interplanetary matter in the vicinity of the Moon during April and May 1966 was recorded by means of satellite Luna-10. Future experiments however must show whether it is characteristic of the Moon halo or is the result of the Moon's passage through some aggregation of dust particles.

Unfortunately, data recording on satellite Luna-12, from October 12 to December 7,1966 , was carried out mostly during meteor showers. During our observations the satellite was in the parts of the orbit shielded from the showers for a very short time, and it was not enough to consider this experiment as the proof of the validity or invalidity of the hypothesis of the existence of the Lunar dust halo.

To find out the regularities in spatial distribution of meteoritic matter, data relating 
to different regions should be accumulated systematically. As to the mass spectrum of particles, its precise determination will become feasible when, with other measurements, the particle velocity is determined.

In our judgment, the obtaining of meteoritic particle samples from large heights, and the determination of their physical properties and chemical composition, is a very topical as well as a very difficult task. At the same time these experiments will be an important contribution to accumulating statistical data about space density of meteoric matter. Many scientists all over the world are working on finding the solution to these problems. Their joint efforts will make it possible to understand much concerning the solid component of interplanetary matter which at present is unknown or insufficiently known to us.

\section{References}

Bohn, J.L., Alexander, W.M., Simmons, W.F. (1967a) Paper presented at Tenth COSPAR Meeting, London.

Bohn, J.L., Alexander, W.M., Weaver, A. (1967b) Paper presented at Tenth COSPAR Meeting, London.

Dubin, M., Alexander, W.M., Berg, O.E. (1963) Smithson. Contr. Astrophys., 7, 109.

Hemenway, C.L., Hallgren, D.S., Kerridge, J.F. (1967) Paper presented at Tenth COSPAR Meeting, London.

Nilsson, C.S. (1966) Science, 153, 1242.

Wlochowicz, R. (1966) Can. J. Phys., 44, 1.

\section{DISCUSSION}

Dohnanyi: What was the threshold sensitivity, with respect to mass, of the Soviet deep-space satellite which failed to detect the Earth's dust cloud at the distances of the order of the Earth radius?

Nazarova: The threshold sensitivity was $10^{-9} \mathrm{gm}$.

Dohnanyi: What average impact velocity did you assume for a meteoroid near the Moon impacting on the Soviet (Luna-10) satellite?

Nazarova: In the reductions of the satellite Luna-10 data we assume $V=15 \mathrm{~km} / \mathrm{sec}$.

Levin: What heights are assumed for the suggested terrestrial dust cloud?

Nazarova: It should be noted that some scientists do not mean the same thing by the term 'Earth dust halo'. In particular, there is no single opinion as to the altitude where this is to be found. That is why it is reasonable, before deciding whether or not the Earth dust halo exists, to agree about the distance of the dust halo from the Earth.

Fedynskij: Perhaps for the definition of the term 'dust cloud', it is better to establish first the value of the space-meteoroid density at the limits of this cloud.

Nazarova: It seems to me that it is necessary to establish the dust cloud distance from the Earth.

Lindblad: Are your microphone sensors acoustically isolated from the satellite body?

Nazarova: In some flights our sensors were isolated acoustically from the satellite body, and in some flights they were not isolated.

Lindblad: How is the piezo-electric crystal mounted to the membrane? It is well known that the risk of spurious counts is considerably reduced if one uses an acoustically isolated membrane with an epoxy glued piezo-electric crystal.

Nazarova: In the case of both isolated and non-isolated detectors the piezo-electric crystal was glued to a sensitive plate with epoxy glue. The isolated detectors were mounted outside the satellite body. 